\title{
Evaluation of a large dish-type concentrator solar lighting system for
}

\author{
underground car park \\ Duofu Song ${ }^{1,2}$, Hui Lv ${ }^{1,2, *}$, Jun Liao ${ }^{1,2}$, Wenjuan Huang ${ }^{1,2}$, Sihao Huang ${ }^{1,2}$, Chunfu Cheng ${ }^{1,2}$, \\ Huiliang Zhou ${ }^{1,2}$,Yuehong $\mathrm{Su}^{3}$, Saffa Riffat ${ }^{3}$ \\ ${ }^{1}$ Hubei Collaborative Innovation Center for High-efficiency Utilization of Solar Energy, Hubei \\ University of Technology, Wuhan 430068, P. R. China \\ ${ }^{2}$ School of Science, Hubei University of Technology, Wuhan 430068, P. R. China \\ ${ }^{3}$ Department of Architecture and Built Environment, University of Nottingham, Nottingham NG7 \\ $2 R D, U K$ \\ *Corresponding author: simonlv76@gmail.com
}

\begin{abstract}
To utilize solar energy more efficiently and reduce lighting power consumption in underground public spaces such as car park, a large dish-type concentrator solar lighting system is put forward along with its evaluation, which is a unique design to apply a laminated layer of beam split thin film coating and thin film solar cells onto the dish reflector. The collected sunlight is split into two parts, one being reflected into a fibre optical bundle (FOB) and transmitted for daylighting while the rest being absorbed by solar cells for electricity generation as other way to replenish daylighting. A set of four solar lighting system using $3.28 \mathrm{~m}$ diameter dish are designed to meet the lighting requirement in a $1771 \mathrm{~m}^{2}$ underground car park. A mathematical model is adopted to calculate the output power and conversion efficiency of solar cells distributed on the parabolic dish surface. The indoor illuminance distribution is given by lighting simulation. The results indicate that the average daylight illuminance in the car park can vary between $62.7 \mathrm{~lx}$ and $284 \mathrm{~lx}$ on 25 February 2016, and between 62.7 lx and 353 lx on 17 August 2016 for two chosen days respectively. For the presented design, the electricity produced by solar cells is just enough to power light emitting diodes (LEDs) for lighting meeting a criterion at night. Considering about $19 \%$ conversion efficiency of solar cells and the efficacy of $129.5 \mathrm{~lm} / \mathrm{W}$ of LEDs, the hybrid solar lighting system can have about $40 \%$ utilization ratio of solar energy, so it can be concluded that a sufficient lighting provision can be provided by the proposed large dish-type concentrator solar lighting system for applications in underground car park.
\end{abstract}

Keywords : Fibre optic bundle daylighting; Beam split thin film; Dish-type concentrator; Thin film solar cells; Underground car park

\section{Introduction}

With fast global development, the contradiction between the demand and the supply of energy has become sharper. To address the associated challenge of climate change, most countries in the world have come together to sign Paris Climate 
Agreement [1], which has seen that the solar energy is playing a more and more important role in energy structure. In developed countries, $20-40 \%$ of gross energy consumption is used for buildings [2, 3], 12-22\% of which is for lighting [3]. There are some underground public places in our daily life such as underground car parks, tunnels and basements, however, being in badly need of lighting all the time as a result of being not sunlit directly, which contributes to a greater energy consumption. And public lighting is an important part of current energy consumption [4].

Direct daylighting from sunlight [5-7] is an important way to solve these problems. Sunlight optical fibre lighting was proposed in 1980 [12], which has become an important way of implementation [8-11]. However, on account of its instability due to the weather and sun illumination time, its development has been restricted. A breakthrough of solution to this problem is required to increase the acceptance and utilization of sunlight and maintain the stability of system. The system presented in this paper can greatly obtain and concentrate solar radiation by applying a large dish [13]. Solar dish systems are applied widely to collect solar radiation for photovoltaic $[14,15]$ and thermal energy employing [16-19] as a result of their high concentrated radiation intensity $[20,21]$ to make solar energy utilized efficiently [14, 22].

By the surface of dish concentrator coated with beam split thin film [23] and coupled with thin film solar cells [24], accounting for $38.9 \%$ of the total radiant energy of sunlight, the visible (VIS) light (400-760nm) [25] is split into two equal parts by beam split thin film, of which the first 50\% VIS light beam is reflected and concentrated onto a secondary reflector and then reflected into FOB, while the rest $50 \%$ VIS light and other spectral radiation (300-400nm and 760-1800nm) will be transmitted through the beam split thin film and absorbed and transformed into electricity by thin film solar cells underneath the beam split thin film. The generated electricity is stored and supplied to the complementary LEDs lighting [26], so as to improve the overall utilization of sunlight. The FOB mentioned is a bundle of optical fibre made of polymethyl methacrylate (PMMA) material with 200dB/km [27] attenuation coefficient.

In this article, the output power and conversion efficiency of solar cells will be calculated from the spectra of sunlight meanwhile the utilization ratio of solar energy, the optical efficiencies of dish concentrator and FOB will be also defined and given. The values of luminous flux from both FOB daylighting and LED lighting will be used to simulate and analyze the application of this system for lighting of an underground car park in Wuhan, China, as illustrated in Figure 1, to prove the system can light that place up to lighting standard efficiently and have a performance of stable illuminance. Furthermore, self-sufficiency of the complementary solar lighting for a long period in a whole year will be analyzed. 


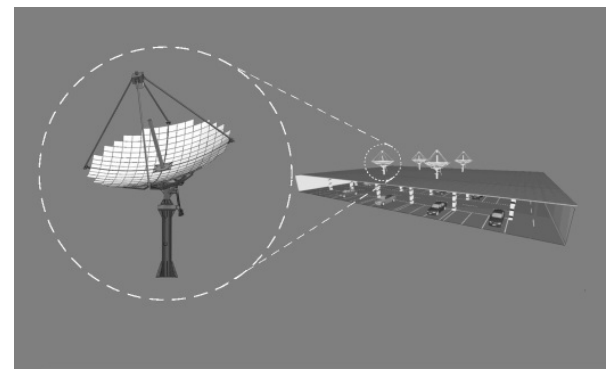

(a)



(b)

Figure 1: Schematic of the lighting system: (a) installation on the grass roof of an underground car park; (b) interior view of daylight illumination in the underground car park.

\section{Description}

The main body of the dish-type concentrator is a primary parabolic dish reflector as illustrated in Figure 2(a). A typical Cassegrain optics structure [28] can be presented with a confocal secondary hyperbolic dish reflector which is used to reflect light into FOB. The optical schematic diagram is given in Figure 2(b). The concentrator is supported by a sun tracking system with four sensors on the quadrants of a bar on the back of the secondary reflector to detect the direction of the sunlight, so it can have a real-time track of the sun in practice and guarantee the system to collect the maximum amount of solar radiation by controlling the rotation of motors [12]. The reflectance of primary reflector is dependent of beam split thin film, and the reflectance $R_{s}(\%)$ of $85 \%$ is assumed for the secondary reflector. Based on our design analysis, the chosen geometries of the primary and secondary reflectors such as diameter, focal distance, half field angle and eccentricity are given in Table 1.

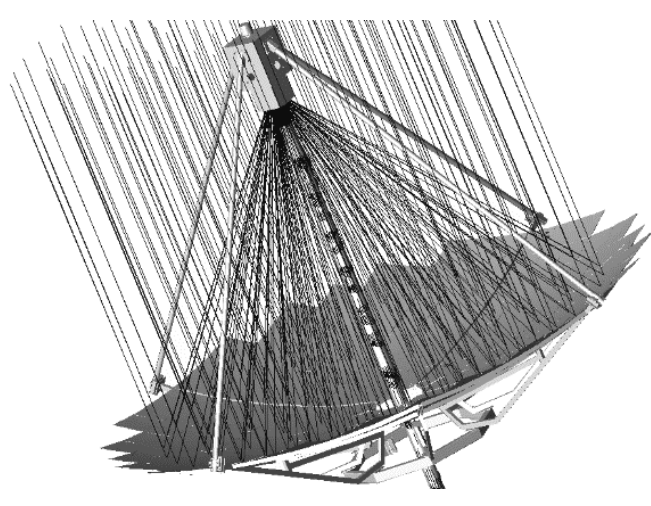

(a)

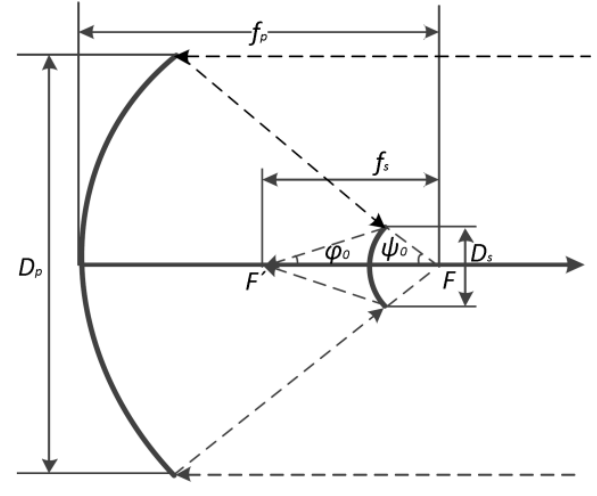

(b)

Figure 2: (a) the structure of dish concentrator; (b) the optical schematic diagram of Cassegrain structure.

The optical surface of the primary reflector is divided into an array of 204 elements [29] and the area of each elemental reflector is about $432.64 \mathrm{~cm}^{2}(20.8 \times$ $20.8 \mathrm{~cm}$ ) for a single solar cell. As for each element, its area and curvature are small, so an identical structure can be used, which can help to reduce the manufacturing 
difficulty and cost of coating film effectively. This measure can also decrease the deformation of the thin film solar cells and facilitate fitting a curved glass dish. The array of elementary reflectors and the structure of solar cell module are shown in Figure 3(a), where a beam split thin film is coated on a curved glass, which is then laminated with a thin film solar cell and a circuit layer to form a module with its edge sealed with butyl glue. Each solar cell module element is fixed in a array parabolic dish panes to from large dish reflector, which are jointed with the cross metal skeleton, and the cross metal skeleton support four metal bars to fix secondary hyperbolic dish reflector. Whole the large dish concentrator is supported with a metal structure as shown in Figure 2(a).

The described beam split thin film [30, 31] is a 13-layer film system made up of $\mathrm{ZnS}$ and $\mathrm{MgF}_{2}$, and it can split VIS light into two equal parts, of which one part is reflected and the other is transmitted and absorbed. The beam split film can meanwhile realize anti-reflection of ultraviolet (UV) light (300-400nm) and infrared (IR) light (760-1800nm) and eliminate or decrease the composition of these light in the concentrated light, which is transmitted through FOB into a room. By removing ultraviolet and infrared light, the concentrated light will be much less harmful to human body [25], have much less thermal effect in the coupling pot of FOB and thus slow down the aging speed of fibres, which can help to maintain the basic form and performance of FOB. When a beam of sunlight strikes on a parabolic dish, the incidence angle are different at different positions of a dish. So it is necessary to analyze the influence of incidence angle on the spectral reflectance $R(\lambda)$ (\%) of beam split thin film by the TFCalc ${ }^{\mathrm{TM}}$ software. From Figure 3(b), we can find that although the reflectance band window slightly shifts by about 40-50nm towards the short-wave direction when the incidence angle changes from $0^{\circ}$ to $30^{\circ}$, the basic shape of reflectance curve remains almost unchanged and had little effect on the actual spectroscopic effect, therefore we used the $R(\lambda)$ values for $0^{\circ}$ incidence angle in calculation in this paper.

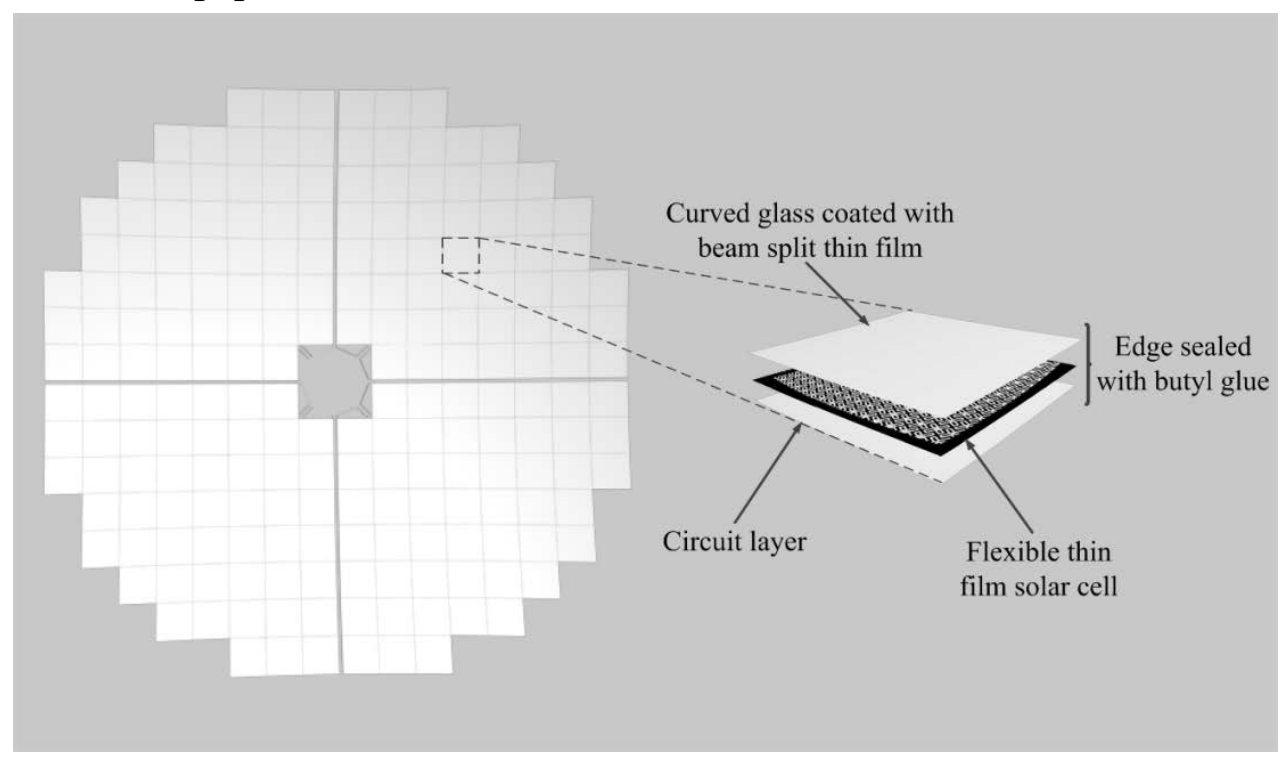


(a)

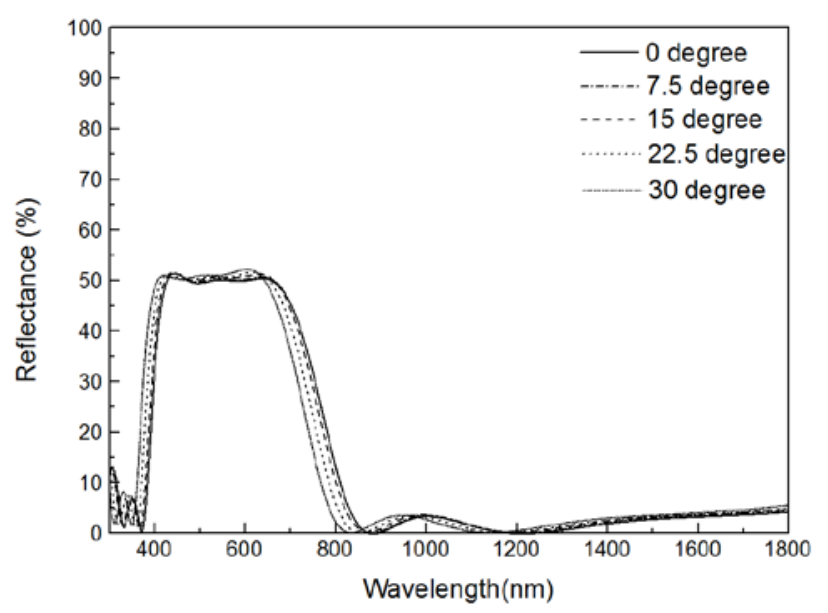

(b)

Figure 3: (a) the beam split thin film and solar cell on the primary reflector; (b) the influence of incidence angle on the reflectance of beam split thin film.

The chosen thin film solar cells are the copper indium gallium diselenide (CIGS) thin film solar cells [32-34], which has good flexibility. Its external quantum efficiency $\operatorname{EQE}(\lambda)(\%)$ is shown in Figure 4.



Figure 4: The spectral EQE (external quantum efficiency) of the CIGS (copper indium gallium diselenide) thin film solar cells [32].

The FOB in this system needs to be light, soft, resistant to bending, and must be also cheap. The plastic optical fibre becomes the best candidate for this design as a result of its low optical loss, suitability for coupling with the light sources and good flexible characteristics. In fact, we chose PMMA as the material of FOB. While the core diameter of single optic fibre is $14 \mathrm{~mm}$, its numerical aperture (NA) is 0.51 [27]. The FOB is composed of 36 optic fibres, with a circular component of $92 \mathrm{~mm}$ diameter. 
The terminal lamps consist of 3 optical fibres, 4 LEDs and diffusers. With the advantages of high optical efficacy, energy conservation, environment protection, long service and cheap price, LED lighting has become a development trend of the future lighting. The choice of LEDs in this design is XLamp XP-G3 of Cree, with its max efficacy of $190 \mathrm{~lm} / \mathrm{W}$ under the binning condition, the rated power of $6 \mathrm{~W}$, and the luminous flux output of $777 \mathrm{~lm}$, and it will supply lighting in cloudy weather and at night. The emergent luminous flux of lamps reaches $2625.6 \mathrm{~lm}$ when only 4 LEDs work, which means it can have very good lighting effect while saving energy. The VIS band of sunlight is guided into the lamps by FOB in daytime. The emergent angle of divergence of optical fibre $2 \alpha$ is determined by $\alpha=\arcsin (\mathrm{NA})$ and it is $61.3^{\circ}$ in this design, but it don't conform to the uniform requirements. A diffuser can solve such problem and it may be based on polycarbonate material with $88 \%$ transmittance. The refraction, reflection, and scattering of light between chemical particles and resin form the uniform effect of light, and not only increase the illuminated area but also decrease the occurrence of dazzling when applied in underground car parks.

As illustrated earlier in Figure 1, the complementary sunlight and electric lighting system is based on the combination of a solar optical fibre daylighting device and electric LED lamps. The former mainly includes a primary dish reflector laminated with thin film solar cell and beam split thin film, a secondary reflector, FOB and a sun-tracking device. The beam split thin film is coating on the curved glass, and the curved glass is laminated with thin film solar cells. When sunlight irradiates beam split thin film, UV, IR and 50\% VIS light are transmitted through the beam split thin film, and are absorbed and transformed into electricity by thin film solar cells underneath, while the other $50 \%$ VIS light is reflected and concentrated to the secondary reflector, and then reflected again and coupled into the FOB, being transmitted to the lamps eventually. The LEDs in lamps are supplied by battery whose power is from the thin film solar cells. If there is power shortage, the battery will get extra power supply from the grid. The LEDs will be controlled with photoswitch to provide light on cloudy weather or at night for complementary lighting.

\section{Results and analysis}

For the calculation of the output power and conversion efficiency of solar cells and the simulation of lighting in underground car park, we chose Wuhan, China as a location to gather outdoor irradiance $E_{e}\left(\mathrm{~W} / \mathrm{m}^{2}\right)$ and illuminance data. Because the data are affected markedly by the seasonal fluctuation, while 2 typical days on February 25, 2016 and August 17, 2016 were chosen, we measured and calculated the hourly averaged values between $300-1800 \mathrm{~nm}$ wavelength $\lambda(\mathrm{nm})$ for the outdoor irradiance, see Figure 5(a).

Because the measured and averaged outdoor irradiance is an integrated for a spectral range, it's necessary to use this hourly outdoor irradiance to give a predicted spectral irradiance $E_{e}(\lambda)\left(\mathrm{W} /\left(\mathrm{m}^{2} \cdot \mathrm{nm}\right)\right)$ according to the normalized spectral irradiance $E_{e}{ }^{\prime}(\lambda)\left(\mathrm{W} /\left(\mathrm{m}^{2} \cdot \mathrm{nm}\right)\right)$, as shown in Figure 5(b) under the condition of air 
mass $A M=1.5$. The spectral irradiance $E_{e}(\lambda)\left(\mathrm{W} /\left(\mathrm{m}^{2} \cdot \mathrm{nm}\right)\right)$ is represented by

$$
E_{e}(\lambda)=I E_{e}^{\prime}(\lambda)
$$

where $I$ is proportionality coefficient, which can be calculated from

$$
I=\frac{E_{e}}{\int_{300}^{1800} E_{e}^{\prime}(\lambda) d \lambda}
$$

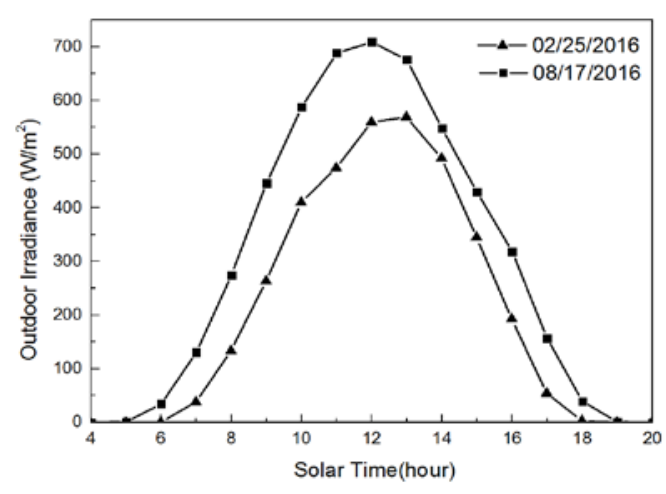

(a)

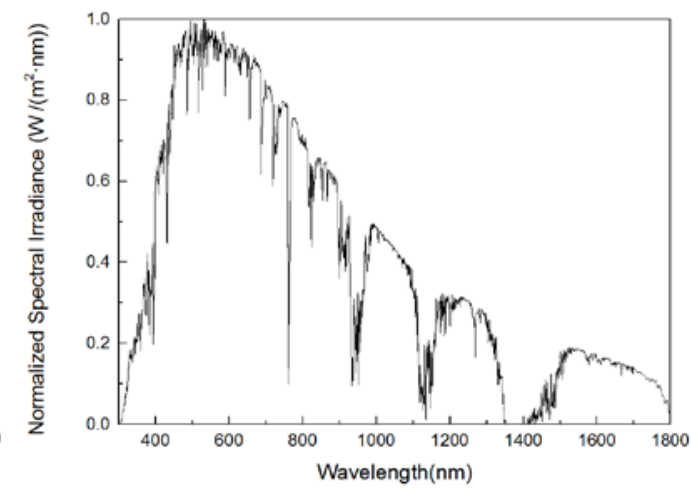

(b)

Figure 5: The solar irradiance: (a) the hourly averaged value of outdoor irradiance between 300-1800nm for 2 days; (b) the normalized spectral irradiance between 300-1800nm under the condition of AM1.5 [35].

When it comes to calculating the output power $P_{\text {out }}(\mathrm{W})$ and conversion efficiency $\eta$ (\%) of solar cells in this system, the spectral irradiance transmitted through the beam split thin film and absorbed by the solar cells is represented as $E_{e}(\lambda, \theta)\left(\mathrm{W} /\left(\mathrm{m}^{2} \bullet \mathrm{nm}\right)\right)$ and it can be given as

$$
E_{e}(\lambda, \theta)=E_{e}(\lambda)[1-R(\lambda)] \cos \theta
$$

Where $\theta$ is the incidence angle of sunlight to solar cells (see Figure 6), and $R(\lambda)$ is the spectral reflectance of beam split thin film given in Figure 3(b).

The short-circuit current density $J_{s c}(\theta)\left(\mathrm{A} / \mathrm{m}^{2}\right)$ of solar cells can be calculated from [36]:

$$
J_{s c}(\theta)=\frac{q}{h c} \int_{300}^{1800} \lambda E_{e}(\lambda, \theta) E Q E(\lambda) d \lambda
$$

Where $q=1.602 \times 10^{-19} \mathrm{C}, \quad h=6.626 \times 10^{-34} \mathrm{~J} \cdot \mathrm{s}$ and $c=2.998 \times 10^{8} \mathrm{~m} / \mathrm{s}$ are electron charge, Planck constant and speed of light. So the short-circuit current $I_{s c}(\mathrm{~A})$ is 


$$
I_{s c}=\iint_{\Sigma} J_{s c}(\theta) d S_{c}
$$

Where $\Sigma$ represents integration over the curved surface of parabolic dish and $d S_{c}$ $\left(\mathrm{m}^{2}\right)$ is infinitesimal of curved surface area of parabolic dish. The relationship between infinitesimal of aperture area of parabolic dish $d S_{p}\left(\mathrm{~m}^{2}\right)$ and $d S_{c}$ is shown in Figure 6 and it can be given as

$$
d S_{p}=d S_{c} \cos \theta
$$

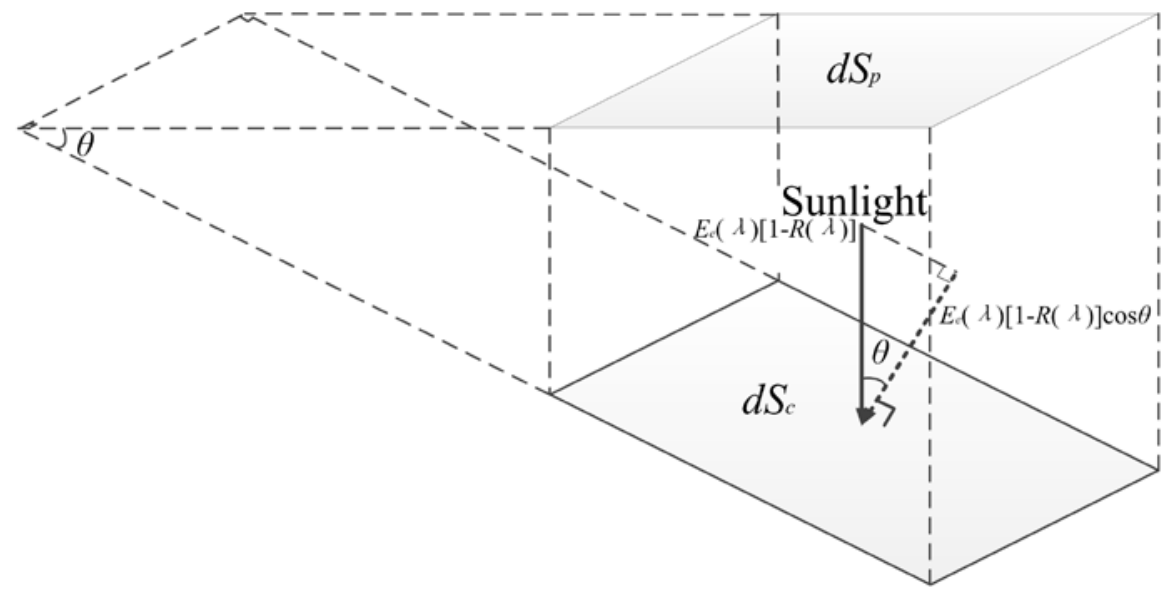

Figure 6: The model of incidence of sunlight and infinitesimal area projection relationship between $d S_{p}$ and $d S_{c}$.

Therefore short-circuit current can be calculated from Equations (3) to (6) as

$$
I_{s c}=\frac{q S_{p}}{h c} \int_{300}^{1800} \lambda E_{e}(\lambda)[1-R(\lambda)] \operatorname{EQE}(\lambda) d \lambda
$$

As for open-circuit voltage $V_{o c}(\mathrm{~V})$, it can be given as [37]:

$$
V_{o c}=\frac{k T}{q} \ln \left(\frac{I_{s c}}{I_{0}}+1\right)
$$

Where $k=1.381 \times 10^{-23} \mathrm{~J} / \mathrm{K}$ is Boltzmann constant and $T(\mathrm{~K})$ is the temperature in Kelvin which is $298 \mathrm{~K}$ under the standard condition. As for saturation current density $I_{0}\left(\mathrm{~A} / \mathrm{cm}^{2}\right)$, it can be calculated from

$$
I_{0}=1.5 \times 10^{5} e^{-\frac{E_{g}}{k T}}
$$

Where $E_{g}(\mathrm{eV})$ is band gap and it is $1.02 \mathrm{eV}$ [38]. Normalized open-circuit voltage $v_{o c}(\mathrm{~V})$ is defined as 


$$
v_{o c}=\frac{V_{o c}}{k T / q}=\frac{q V_{o c}}{k T}
$$

and fill factor FF is followed as

$$
F F=\frac{v_{o c}-\ln \left(v_{o c}+0.72\right)}{v_{o c}+1}
$$

Therefore output power can be calculated from

$$
P_{\text {out }}=V_{o c} I_{s c} F F
$$

The conversion efficiency can be given as

$$
\eta=\frac{P_{\text {out }}}{P_{\text {in }}}
$$

As for incident optical power $P_{\text {in }}(\mathrm{W})$ of solar cells in this system, it can be expressed as

$$
P_{\text {in }}=S_{p} \int_{300}^{1800} E_{e}(\lambda)[1-R(\lambda)] d \lambda
$$

The hourly averaged output power and conversion efficiency are shown in Figure 7, where were calculated for one dish concentrator system between 4 and 20 o'clock on 2 example days, respectively.



(a)

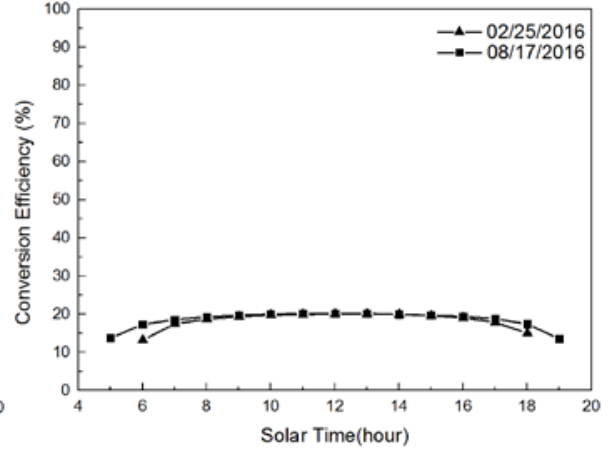

(b)

Figure 7: The output of solar cells for one dish concentrator: (a) the hourly averaged output power; (b) the hourly averaged conversion efficiency.

As for simulation of this system applied to an underground car park, which was built in software with the geometries of $52.4 \mathrm{~m}$ long, $33.8 \mathrm{~m}$ wide and $3.8 \mathrm{~m}$ high. The reflectance of car park ceiling, wall and floor are $70 \%, 90 \%$ and $22.3 \%$, respectively. There are 60 parking spaces and each parking area is $12.72 \mathrm{~m}^{2}(5.3 \times 2.4 \mathrm{~m})$ [39], which is denoted in Figure 8(a). Each dish concentrator FOB daylighting system supports 12 lamps. As is illustrated in Figure 8(b), four systems are assumed installed on the roof of the underground car park and lead to the 48 lamps distributed in an array of $6 \times 8$ array at a height of $3.5 \mathrm{~m}$ from the floor. Due to their different distance from the dish, 48 lamps are divided into 4 types according to the FOB length, and 
labelled as No.1, No.2, No.3 and No.4. Their number and length are shown in Table 2.

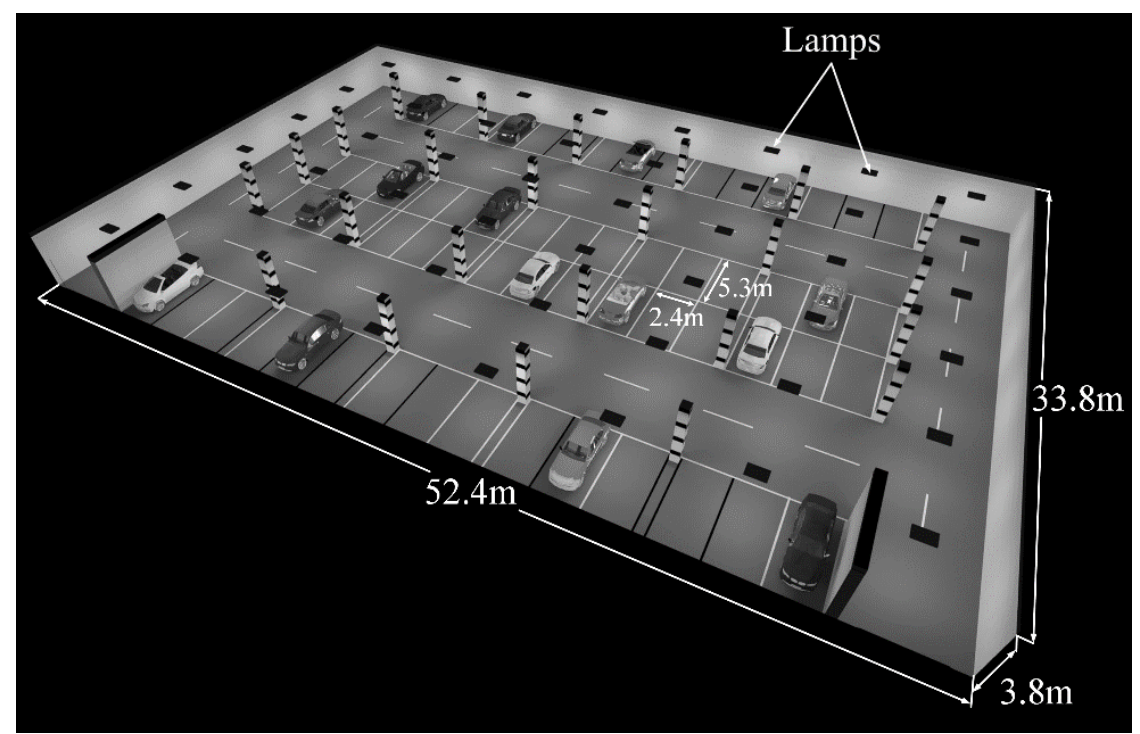

(a)

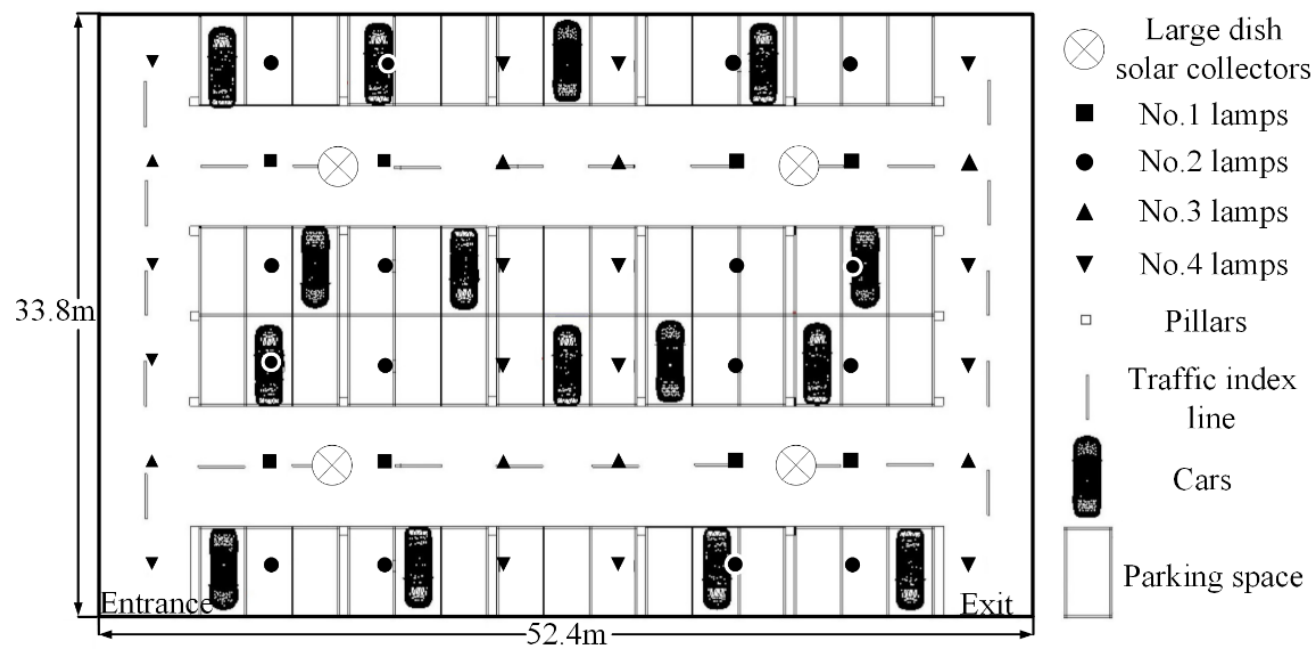

(b)

Figure 8: The underground car park: (a) the optical model of the underground car park built in the software; (b) the distribution of 48 lamps.

The luminous flux at lamps can be deduced for one system from outdoor irradiance through FOB to lamps. Transmission efficiency of FOB is determined by attenuation coefficient, which is shown in Figure 9 for the attenuation coefficient $A(\lambda)(\mathrm{dB} / \mathrm{km})$ of PMMA optical fibre. Attenuation coefficient can be calculated in decibels from [40]

$$
A(\lambda)=\frac{10}{L} \lg \frac{\Phi_{e i}(\lambda)}{\Phi_{e o}(\lambda)}
$$


Where $L(\mathrm{~km}), \Phi_{e i}(\lambda)(\mathrm{W} / \mathrm{nm})$ and $\Phi_{e o}(\lambda)(\mathrm{W} / \mathrm{nm})$ are length of FOB, input spectral radiation flux and output spectral radiation flux. Because the length of FOB is long relatively, it becomes the main factor of light attenuation.

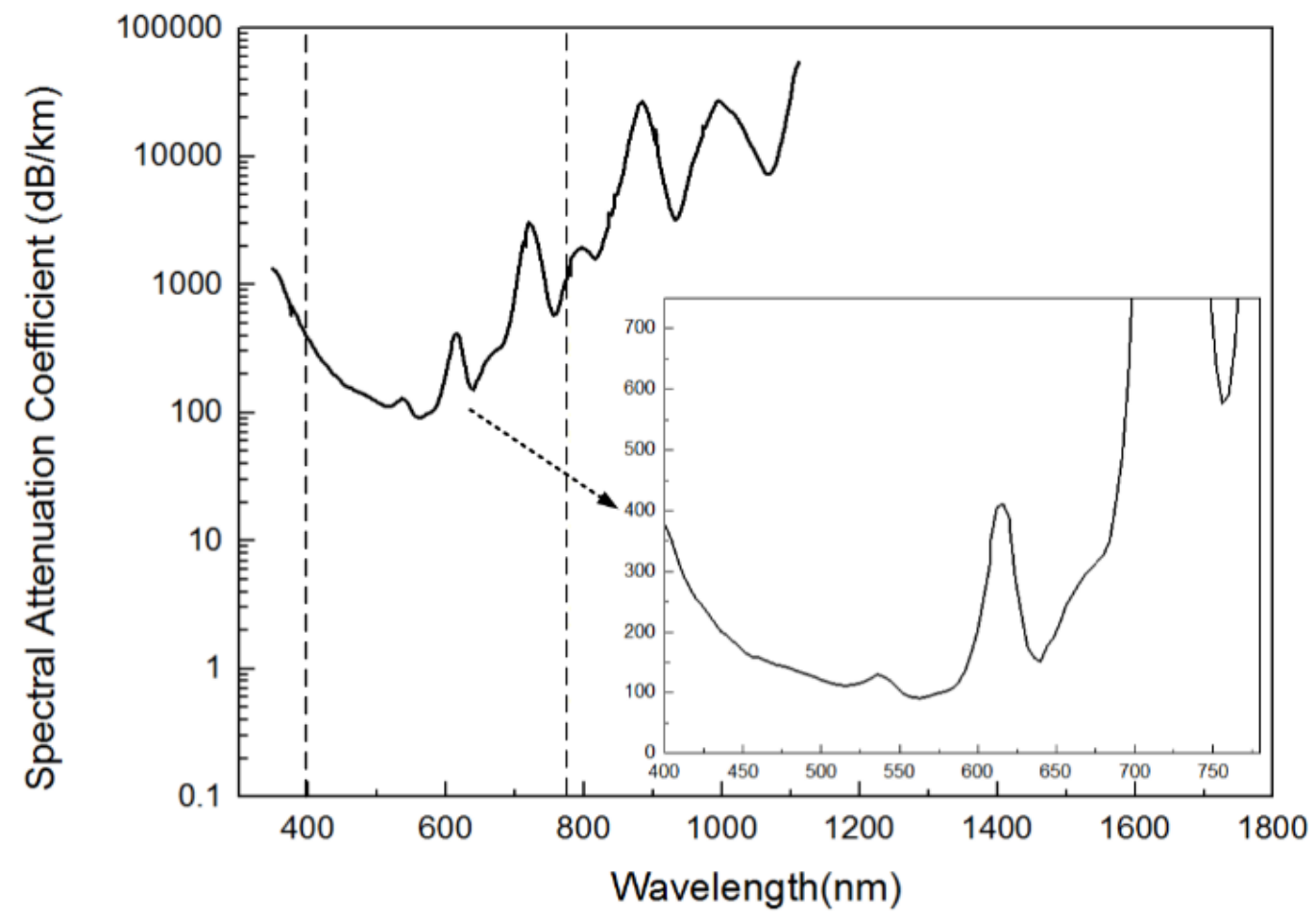

Figure 9: The attenuation coefficient of PMMA optical fibre [41].

Transmission efficiency of FOB $\eta(\lambda)(\%)$ is

$$
\eta(\lambda)=\frac{\Phi_{e o}(\lambda)}{\Phi_{e i}(\lambda)}
$$

So the transmission efficiency of FOB can be given from Equations (15) and (16) as

$$
\eta(\lambda)=\frac{1}{10^{\frac{A(\lambda) L}{10}}}
$$

While one system supports 12 lamps, for every lamp, the input spectral radiation flux is related by

$$
\Phi_{e i}(\lambda)=\frac{1}{12} S_{p} E_{e}(\lambda) R(\lambda) R_{s}
$$

So the output spectral radiation flux is calculated from

$$
\Phi_{e o}(\lambda)=\frac{1}{12} S_{p} E_{e}(\lambda) R(\lambda) R_{s} \eta(\lambda)
$$

What are mentioned above is radiometry, but photometry is needed for the simulation of daylighting in software. The relationship between radiometry and photometry in this paper are represented by luminous efficacy $K(\lambda)(\mathrm{lm} / \mathrm{W})$ and spectral luminous 
efficiency of photopic vision $V(\lambda)$ (see Figure 10) as follow:

$$
\begin{aligned}
& K(\lambda)=\frac{\Phi_{v}(\lambda)}{\Phi_{e o}(\lambda)} \\
& V(\lambda)=\frac{K(\lambda)}{K_{m}}
\end{aligned}
$$

Where $\Phi_{v}(\lambda)(\mathrm{lm} / \mathrm{nm})$ and $K_{m}(\mathrm{~lm} / \mathrm{W})$ are spectral luminous flux and the maximum luminous efficacy, and $K_{m}$ is $683 \mathrm{~lm} / \mathrm{W}$.

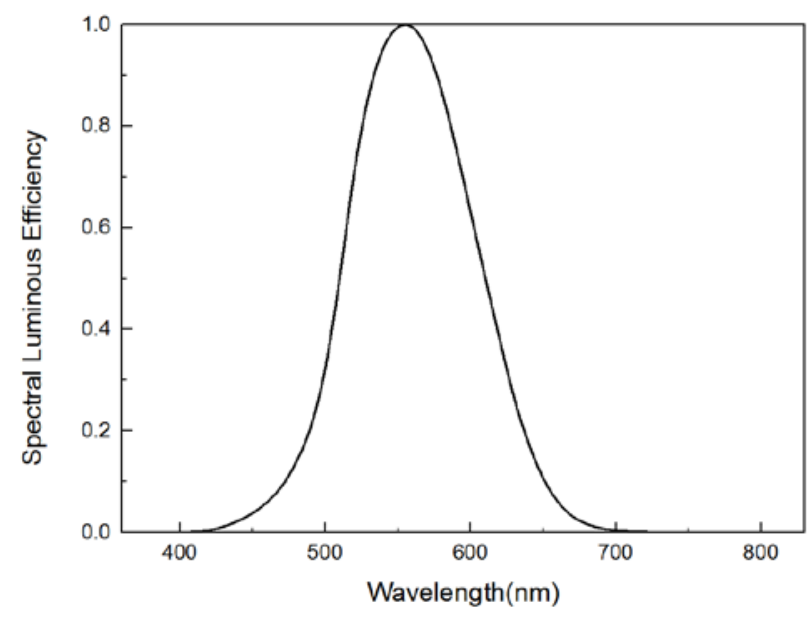

Figure 10: The spectral luminous efficiency of photopic vision [42].

The spectral luminous flux is given from Equations (20) and (21) as

$$
\Phi_{v}(\lambda)=K_{m} V(\lambda) \Phi_{e o}(\lambda)
$$

Synthesizing (2), (17), (19) and (22), luminous flux $\Phi_{v}(\mathrm{~lm})$ is calculated from

$$
\Phi_{v}=\frac{E_{e} S_{p} R_{s} K_{m}}{12 \int_{300}^{1800} E_{e}{ }^{\prime}(\lambda) d \lambda} \int_{300}^{1800} \frac{V(\lambda) R(\lambda) E_{e}{ }^{\prime}(\lambda)}{10^{\frac{A(\lambda) L}{10}}} d \lambda
$$

It is calculated for one system that the average daylight luminous flux of every hour from outdoor irradiance through FOB to lamps for the 4 types of lamps between 4:00-20:00 for 2 example days on February 25, 2016 and August 17, 2016, respectively, which are given in Figure 11. 


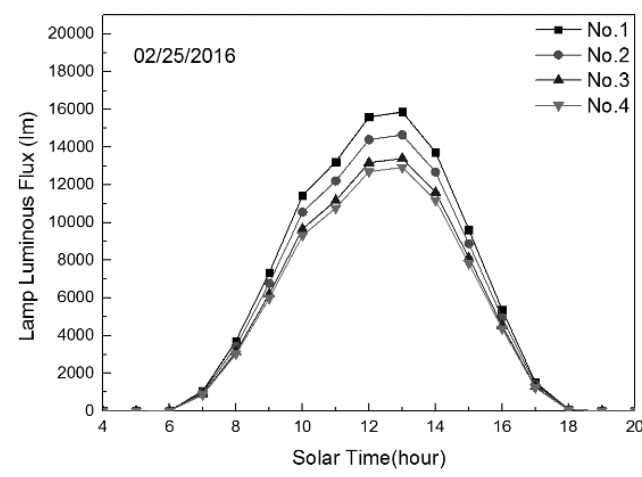

(a)

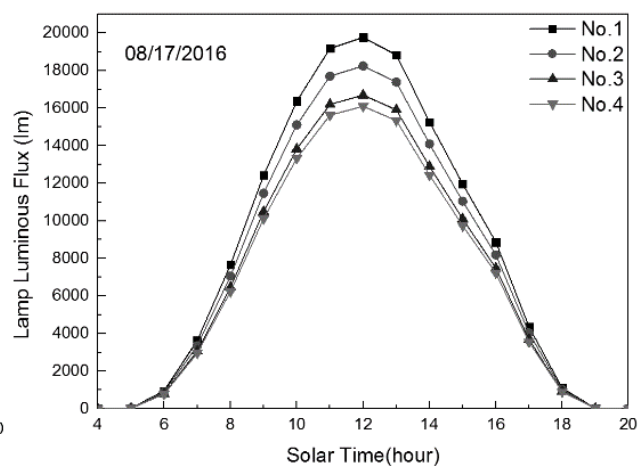

(b)

Figure 11: The hourly averaged luminous flux of daylight for each type lamp: (a) the luminous flux on 02/25/2016; (b) the luminous flux on 08/17/2016.

The optical efficiencies of FOB $\eta_{f}(\%)$ and the optical efficiency of dish concentrator $\eta_{d}$ (\%) can be defined as

$$
\begin{gathered}
\eta_{f}=\frac{\int_{300}^{1800} \Phi_{e o}(\lambda) d \lambda}{\int_{300}^{1800} \Phi_{e i}(\lambda) d \lambda}=\frac{\int_{300}^{1800} E_{e}(\lambda) R(\lambda) / 10^{\frac{A(\lambda) L}{10}} d \lambda}{\int_{300}^{1800} E_{e}{ }^{\prime}(\lambda) R(\lambda) d \lambda} \\
\eta_{d}=\frac{\sum_{i=1}^{i=4} j \int_{300}^{1800} \frac{1}{12} S_{p} E_{e}(\lambda) R(\lambda) R_{s} \frac{1}{10^{\frac{A(\lambda) L i}{10}}} d \lambda}{E_{e} S_{p}}=\frac{R_{s}}{12 \int_{300}^{1800} E_{e}{ }^{\prime}(\lambda) d \lambda} \sum_{i=1}^{i=4} j \int_{300}^{1800} \frac{E_{e}{ }^{\prime}(\lambda) R(\lambda)}{10^{\frac{A(\lambda) L i}{10}}} d \lambda \\
,(i=1, j=2 ; i=2, j=4 ; i=3, j=2 ; i=4, j=4)
\end{gathered}
$$

The numerator of Equation (24) and (25) mean the output radiation which should be integrated from $300 \mathrm{~nm}$ to $1800 \mathrm{~nm}$ theoretically, but in fact, on the one hand, the VIS to near infrared (about 350-1100nm) is the wavelength range in which the attenuation coefficient of PMMA optical fibre is minimum [41] meanwhile that of the rest ultraviolet , infrared and red wavelength range are high [41, 43], on the other hand , the most ultraviolet and infrared sunlight are transmitted through the beam split thin film, and light transmitted by PMMA optical fibre are rare in this wavelength range according to the spectral reflectance $R(\lambda)$ of beam split thin film. Therefore the wavelength range of integral for the numerator of Equation (24) and (25) is simplified to $350-1100 \mathrm{~nm}$ when it is calculated. The Optical efficiencies of FOB are shown in Table 3 and the optical efficiency of dish concentrator is $12.16 \%$.

According to the hourly daylight luminous flux from FOB in each lamp at daytime and the luminous flux of LEDs lighting only in lamps at night, we set values of luminous flux in lighting simulation for every lamp. Lighting is simulated every hour for 2 example days in the underground car park in the software to give the 
illuminance at the level of floor.

The average values of indoor illuminance are shown in Figure 12, indicating a performance of stable illuminance under 353lx and above 62.7lx. According to Standard for lighting design of buildings (GB50034-2013) [44], lighting in underground car parks, taking the floor as reference plane (height is $0 \mathrm{~m}$ ), the recommended illuminance is $50 \mathrm{~lx}$. It can be found from the simulation of 48 lamps supported by 4 FOB daylighting systems in the $1771 \mathrm{~m}^{2}$ underground car park, the sunlight lighting only are for 9 hours and 11hours on these 2 days from Figure 12, and the maximum values of illuminance on the days are $284 \mathrm{~lx}$ and $353 \mathrm{~lx}$ around 13 and 12 o'clock, while the average value of illuminance for using LEDs only at night is set at $62.7 \mathrm{~lx}$. Therefore, not only does the system supply sufficient lighting by using solar radiation at daytime, but also meet the standard of lighting basically for the whole day.

The direct daylight delivered by FOB is sufficient during the most of day time. As for the electricity output of one system on 25 February 2016, the battery was charged about $4.298 \mathrm{kWh}$ and discharged about $4.320 \mathrm{kWh}$ for LEDs, so the electricity produced by solar cells is just enough to power LEDs for lighting at that night, which demands more appropriate spectral reflectance of beam splitting to realize the self-sufficiency of the system.

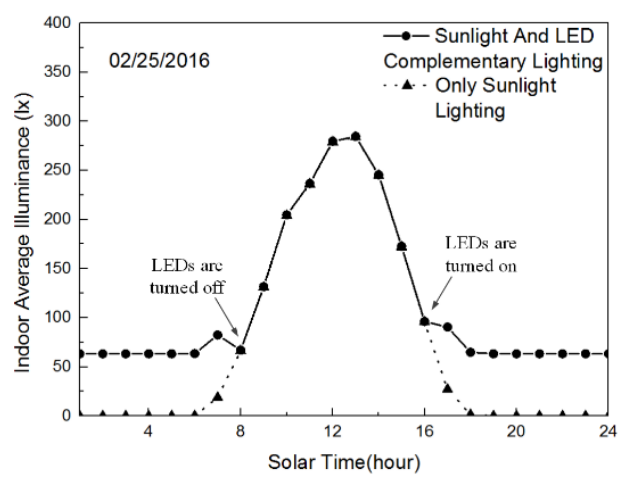

(a)

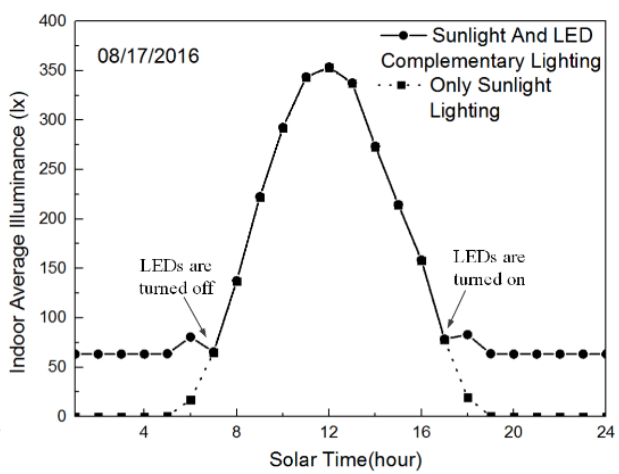

(b)

Figure 12: The hourly averaged values of illuminance measured on the floor in the underground car park: (a) indoor average illuminance on 02/25/2016; (b) indoor average illuminance on 08/17/2016.

As for the uniformity of illuminance in underground car park, it is illustrated in Figure 13 by the distribution of illuminance. Figure 13(a), 13(b) and 13(c) are distribution of illuminance at 13 o'clock on 25 February 2016, at 12 o'clock on 17 August 2016 and by using LEDs only at night. Although there are peak value region around the lamps space, the whole distribution of illuminance is nearly uniform, and we can conclude the distribution of illuminance of all the hours are nearly uniform by the distribution of illuminance of maximum and minimum values of illuminance. 

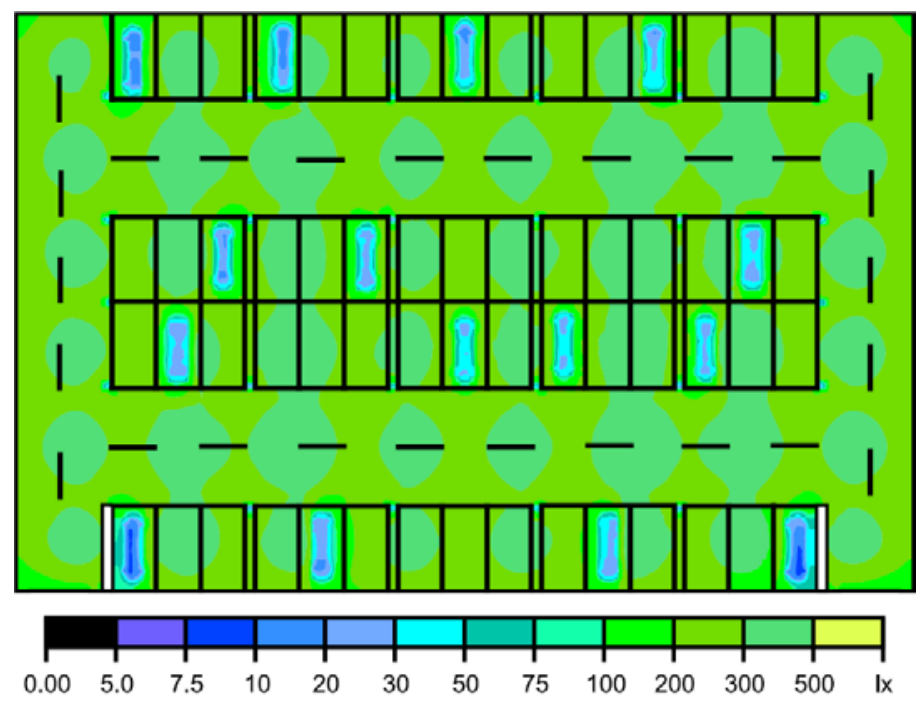

(a)

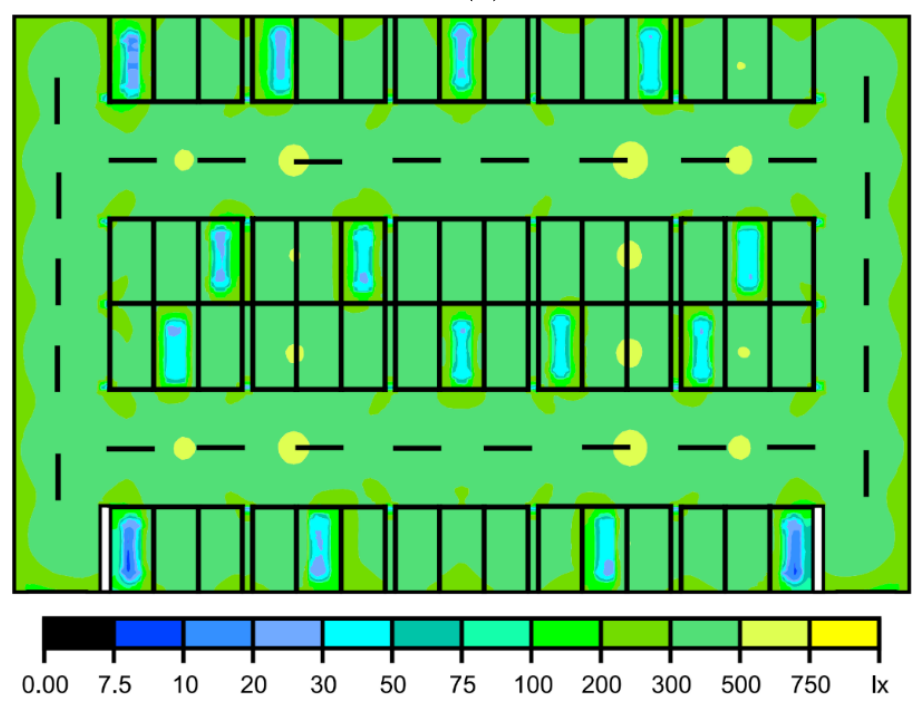

(b)
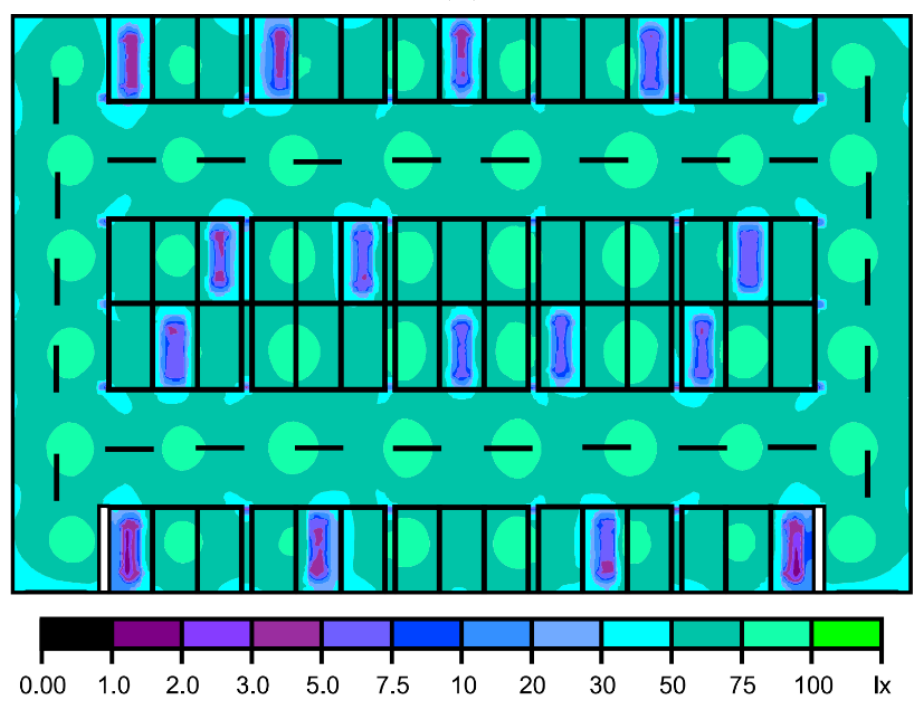

(c)

Figure 13: The distribution of illuminance: (a) daylight illuminance at 13 o'clock on 02/25/2016; (b) daylight illuminance at 12 o'clock on 08/17/2016; (c) the distribution 
of illuminance by using LEDs only at night.

The utilization ratio of solar energy $\sigma(\%)$ of this system should be considered in the two ways of utilization, 50\% VIS for daylighting and the remaining sunlight for electricity generation and then lighting at night. As for the latter, the output power of solar cells is measured by electric power, therefore it's necessary to calculate the equivalent electric power for the direct lighting by the first $50 \%$ VIS light by using the supposed rated efficacy $E(\mathrm{~lm} / \mathrm{W})$ of LED. For a luminous flux $(\mathrm{lm})$ of a LED same as that of sunlight, the equivalent electric power $P_{e}(\mathrm{~W})$ of LED can be given as

$$
P_{e}=\frac{2 \Phi_{v 1}+4 \Phi_{v 2}+2 \Phi_{v 3}+4 \Phi_{v 4}}{E}
$$

Where $\Phi_{v n},(n=1,2,3,4)(\mathrm{lm})$ are luminous flux from the FOB system at the lamps of 4 types, so the utilization ratio of solar energy can be defined as

$$
\sigma=\frac{P_{\text {out }}+P_{e}}{E_{e} S_{p}}
$$

When the efficacy $E=129.5 \mathrm{~lm} / \mathrm{W}$ (the rated efficacy of XLamp XP-G3 of Cree), the system utilization ratio of solar energy every hour can be shown in Figure 14.

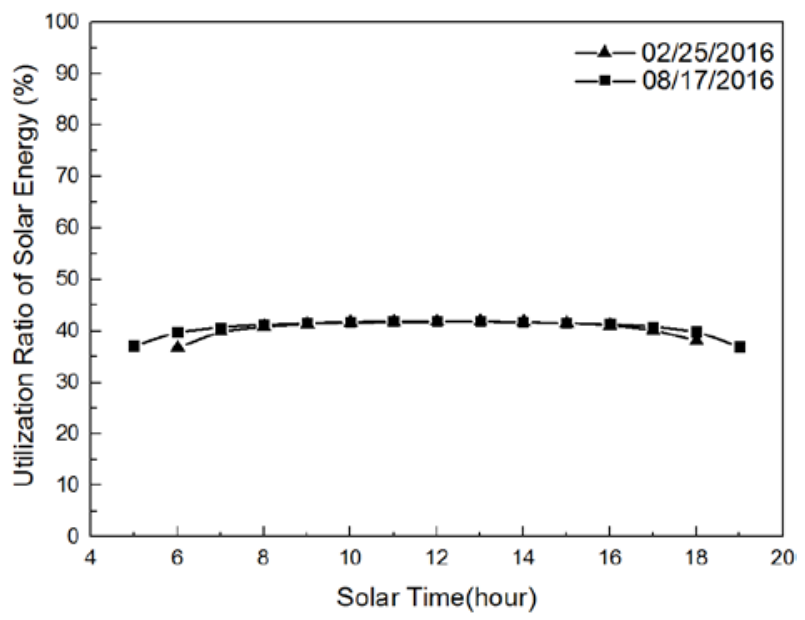

Figure 14: The lighting system's utilization ratio of solar energy at different hours.

\section{Conclusions}

A large dish-type concentrator solar lighting system has been described and evaluated for lighting in an underground car park.

The beam split thin film has been selectively designed to reflect part of visible light and meanwhile transmit the rest visible and all invisible light. The proposed structure of large dish can concentrate solar radiation, split spectra and produce electricity as well, by using a laminated layer of beam split coating and thin film solar cell. The conversion efficiency of solar cells is about $19 \%$. As for the transmission of 
sunlight, the optical efficiency of dish concentrator is $12.16 \%$. The simulation of lighting a $1771 \mathrm{~m}^{2}$ underground car park by the large dish-type concentrator solar lighting systems achieves the average daylight illuminance varying between $62.7 \mathrm{~lx}$ which is the setting point for LED light and the maximum 284 lx on 25 February 2016 and between $62.7 \mathrm{~lx}$ and $353 \mathrm{~lx}$ on 17 August 2016, respectively, in Wuhan, China, with about $40 \%$ utilization ratio of solar energy which proves the system can light that place up to lighting standard efficiently and have a performance of stable illuminance.

Furthermore, for the current design to evaluate the overall lighting performance of system, the provision is just enough and meets the standard of lighting basically for the whole day. In the follow-up research, we will try to balance the beam split between daylighting and LEDs lighting for different days, and seek to optimize the most appropriate spectral reflectance in beam splitting to realize the self-sufficiency of the complementary solar lighting for a long period in a whole year.

\section{Acknowledgement}

The authors gratefully acknowledge Hubei Collaborative Innovation Center for High-efficiency Utilization of Solar Energy, Hubei University of Technology, for its Open Foundation under Grant No. HBSKFTD2016001. This work was also supported partly by the National Undergraduate Training Programs for Innovation and Enterpreneurship under Grant No. 201710500008, in part by the International Science \& Technology Cooperation Project of Wuhan Science and Technology Bureau under Grant No. 2017030209020254, and in part by the International Science \& Technology Cooperation Program of China under Grant No. 2016YFE0124300.

\section{References}

[1] Joeri Rogelj, Michel Den Elzen, Niklas Höhne, et al. Paris Agreement climate proposals need a boost to keep warming well below 2 C. Nature, 2016, 534(7609): 631-639.

[2] European Parliament and Council. Directive 2010/31/EU of the European Parliamentand of the Council of 19 May 2010 on the energy performance of buildings.Official Journal of the European Union 2010;L153:13-35.

[3] Luis Pérez-Lombard, José Ortiz, Christine Pout. A review on buildings energy consumption information. Energy and Buildings, 2008, 40(3): 394-398.

[4] Eleonora Riva Sanseverino, Gianluca Scaccianoce, Valentina Vaccaro, et al. Smart city and public lighting Smart city and public lighting. Environment and Electrical Engineering (EEEIC), 2015 IEEE 15th International Conference on. IEEE: 665-670.

[5] Yuanyi Chen, Junjie Liu, Jingjing Pei, et al. Experimental and simulation study on the performance of daylighting in an industrial building and its energy saving potential. Energy and Buildings, 2014, 73: 184-191.

[6] David Lingfors, Tarja Volotinen. Illumination performance and energy saving of a solar fiber optic lighting system. Optics express, 2013, 21(104): A642-A655.

[7] C Sapia. Daylighting in buildings: Developments of sunlight addressing by 
optical fiber. Solar Energy, 2013, 89: 113-121.

[8] Irfan Ullah, Hui Lv, Allen Jong-Woei Whang, et al. Analysis of a novel design of uniformly illumination for Fresnel lens-based optical fiber daylighting system. Energy and Buildings, 2017, 154: 19-29.

[9] Ravi Gorthala, Meg Tidd, Sean Lawless. Design and development of a faceted secondary concentrator for a fiber-optic hybrid solar lighting system. Solar Energy, 2017, 157: 629-640.

[10] Georgios E Arnaoutakis, Jose Marques-Hueso, Tapas K Mallick, et al. Coupling of sunlight into optical fibres and spectral dependence for solar energy applications. Solar Energy, 2013, 93: 235-243.

[11] Xiaodi Xue, Hongfei Zheng, Yuehong Su, et al. Study of a novel sunlight concentrating and optical fibre guiding system. Solar Energy, 2011, 85(7): 1364-1370.

[12] Canan Kandilli, Koray Ulgen, Arif Hepbasli. Exergetic assessment of transmission concentrated solar energy systems via optical fibres for building applications. Energy and Buildings, 2008, 40(8): 1505-1512.

[13] Yong Shuai, Xin-Lin Xia, He-Ping Tan. Radiation performance of dish solar concentrator/cavity receiver systems. Solar Energy, 2008, 82(1): 13-21.

[14] Joe Coventry, Charles Andraka. Dish systems for CSP. Solar Energy, 2017.

[15] Kok-Keong Chong, Tiong-Keat Yew, Chee-Woon Wong, et al. Dense-array concentrator photovoltaic prototype using non-imaging dish concentrator and an array of cross compound parabolic concentrators. Applied Energy, 2017.

[16] Saša R Pavlović, Darko M Vasiljević, Velimir P Stefanović, et al. OPTICAL ANALYSIS AND PERFORMANCE EVALUATION OF A SOLAR PARABOLIC DISH CONCENTRATOR. Thermal Science, 2016, 20.

[17] V Sriram, B Kanimozhi. Design and analysis of reflectivity for Mylar-coated solar dish. International Journal of Ambient Energy, 2016: 1-3.

[18] Az Hafez, Ahmed Soliman, Ka El-Metwally, et al. Design analysis factors and specifications of solar dish technologies for different systems and applications. Renewable and Sustainable Energy Reviews, 2017, 67: 1019-1036.

[19] V Thirunavukkarasu, M Sornanathan, M Cheralathan. An experimental study on energy and exergy performance of a cavity receiver for solar parabolic dish concentrator. International Journal of Exergy, 2017, 23(2): 129-148.

[20] Gang Xiao, Tianfeng Yang, Dong Ni, et al. A model-based approach for optical performance assessment and optimization of a solar dish. Renewable Energy, 2017, 100: 103-113.

[21] Jun Wang, Song Yang, Chuan Jiang, et al. A novel 2-stage dish concentrator with improved optical performance for concentrating solar power plants. Renewable Energy, 2017, 108: 92-97.

[22] Asepta Surya Wardhana, Heri Suryoatmojo, Mochamad Ashari. Design of parabolic solar concentrator to improve the optical efficiency for thermal engine generator using dual reflector Gregorian method Design of parabolic solar concentrator to improve the optical efficiency for thermal engine generator using dual reflector Gregorian method. Intelligent Technology and 
Its Applications (ISITIA), 2016 International Seminar on. IEEE: 457-464.

[23] Ahmad Mojiri, Robert Taylor, Elizabeth Thomsen, et al. Spectral beam splitting for efficient conversion of solar energy - A review. Renewable and Sustainable Energy Reviews, 2013, 28: 654-663.

[24] Adrian Chirilă, Stephan Buecheler, Fabian Pianezzi, et al. Highly efficient $\mathrm{Cu}$ (In, Ga) Se2 solar cells grown on flexible polymer films. Nature materials, 2011, 10(11): 857-861.

[25] Lindsay R Sklar, Fahad Almutawa, Henry W Lim, et al. Effects of ultraviolet radiation, visible light, and infrared radiation on erythema and pigmentation: a review. Photochemical \& Photobiological Sciences, 2013, 12(1): 54-64.

[26] Chih-Hsuan Tsuei, Wen-Shing Sun, Chien-Cheng Kuo. Hybrid sunlight/LED illumination and renewable solar energy saving concepts for indoor lighting. Optics express, 2010, 18(104): A640-A653.

[27] Canan Kandilli, K Ulgen. Review and modelling the systems of transmission concentrated solar energy via optical fibres. Renewable and Sustainable Energy Reviews, 2009, 13(1): 67-84.

[28] Charles E Mauk, H William Prengle, Eddy Chi-Hua Sun. Optical and thermal analysis of a Cassegrainian solar concentrator. Solar Energy, 1979, 23(2): 157-167.

[29] Pierre J Verlinden, Allan Lewandowski, Carl Bingham, et al. Performance and reliability of multijunction III-V modules for concentrator dish and central receiver applications Performance and reliability of multijunction III-V modules for concentrator dish and central receiver applications. Photovoltaic Energy Conversion, Conference Record of the 2006 IEEE 4th World Conference on. IEEE,1: 592-597.

[30] J Kim, Jonathan R Birge, V Sharma, et al. Ultrabroadband beam splitter with matched group-delay dispersion. Optics letters, 2005, 30(12): 1569-1571.

[31] Alexander V Tikhonravov, Michael K Trubetskov, Vladimir V Protopopov, et al. Application of the needle optimization technique to the design of X-ray mirrors Application of the needle optimization technique to the design of X-ray mirrors. Proc SPIE.3738: 248-254.

[32] Js Ward, K Ramanathan, Fs Hasoon, et al. A 21.5\% efficient Cu (In, Ga) Se2 thin - film concentrator solar cell. Progress in Photovoltaics: Research and Applications, 2002, 10(1): 41-46.

[33] Mario Pagliaro, Rosaria Ciriminna, Giovanni Palmisano. Flexible solar cells. ChemSusChem, 2008, 1(11): 880-891.

[34] Gregory Brown, Peter Stone, Jacob Woodruff, et al. Device characteristics of a $17.1 \%$ efficient solar cell deposited by a non-vacuum printing method on flexible foil Device characteristics of a $17.1 \%$ efficient solar cell deposited by a non-vacuum printing method on flexible foil. Photovoltaic Specialists Conference (PVSC), 2012 38th IEEE. IEEE: 003230-003233.

[35] Daryl R Myers, Christian A Gueymard. Description and availability of the SMARTS spectral model for photovoltaic applications Description and availability of the SMARTS spectral model for photovoltaic applications. 
Proceedings of SPIE.5520: 56-67.

[36] Juan M Russo, Deming Zhang, Michael Gordon, et al. Spectrum splitting metrics and effect of filter characteristics on photovoltaic system performance. Optics express, 2014, 22(102): A528-A541.

[37] Martin A. Green. Solar Cells: Operating Principles, Technology and System Applications. The University of New South Wales: Kensington, 1992.

[38] M Venkatachalam, Md Kannan, S Jayakumar, et al. Effect of annealing on the structural properties of electron beam deposited CIGS thin films. Thin Solid Films, 2008, 516(20): 6848-6852.

[39] Ministry of Housing and Urban-Rural Development of the P.R.China. JGJ100-2015Code for design of parking garage building. China Architecture \& Building Press: Beijing, 2015.

[40] C Koeppen, Rf Shi, Wd Chen, et al. Properties of plastic optical fibers. JOSA B, 1998, 15(2): 727-739.

[41] Joseba Zubia, Jon Arrue. Plastic optical fibers: An introduction to their technological processes and applications. Optical Fiber Technology, 2001, 7(2): 101-140.

[42] Ji Hye Oh, Su Ji Yang, Young Rag Do. Healthy, natural, efficient and tunable lighting: four-package white LEDs for optimizing the circadian effect, color quality and vision performance. Light: Science \& Applications, 2014, 3(2): e141.

[43] Toshikuni Kaino. Absorption losses of low loss plastic optical fibers. Japanese journal of applied physics, 1985, 24(12R): 1661.

[44] Ministry of Housing and Urban-Rural Development of the P.R.China. GB50034-2013Standard for lighting design of buildings. China Architecture \& Building Press : Beijing, 2013. 
Table 1: Parameters of the concentrator

\begin{tabular}{lll}
\hline Parameter & Primary reflector & Secondary reflector \\
\hline Diameter & $D_{p}=3.28 \mathrm{~m}$ & $D_{s}=18.6 \mathrm{~cm}$ \\
Area & $S_{p}=8.45 \mathrm{~m}^{2}$ & $S_{s}=271.7 \mathrm{~cm}^{2}$ \\
Focal distance & $f_{p}=1.54 \mathrm{~m}$ & $f_{s}=21.9 \mathrm{~cm}$ \\
Half field angle & $\psi_{0}=56.08^{\circ}$ & $\varphi_{0}=30.66^{\circ}$ \\
Eccentricity & $e_{p}=1$ & $e_{s}=3.12$ \\
\hline
\end{tabular}


Table 2: Parameters of 48 lamps

\begin{tabular}{lll}
\hline Type of lamps & Number & Length of FOB(m) \\
\hline No.1 & 8 & 6.21 \\
No.2 & 16 & 8.9 \\
No.3 & 8 & 12 \\
No.4 & 16 & 13.3 \\
\hline
\end{tabular}


Table 3: Optical efficiencies of FOB

\begin{tabular}{ll}
\hline Length of $\mathrm{FOB}(\mathrm{m})$ & Optical efficiencies of FOB \\
\hline 6.21 & $64.11 \%$ \\
8.9 & $56.41 \%$ \\
12 & $49.29 \%$ \\
13.3 & $46.7 \%$ \\
\hline
\end{tabular}

